\title{
Hand Posture Recognition For Complex DECISION MAKING
}

\author{
Mrs.Ayesha Butalia ${ }^{1}$, Swapnil Patil, Manjiri Bangali, Bhushan Dusane and \\ Tejswini Chaure, Mrs. Maya Ingale. \\ ${ }^{1}$ Department Of Computer Engineering, MIT College Of Engg. , Pune.
}

ayeshabutalia@yahoo.co.in

\begin{abstract}
:-
Gesture recognition can be seen as a way for computers to begin to understand human body language, thus building a richer bridge between machine and humans than primitive text, user interfaces or even GUI's. Gesture recognition method used in this paper aims on recognizing hand postures as well as facial expressions. Here, facial expressions are used as context for the gesture recognized that helps in giving additional information about the gestures performed. Thus the information obtained from this static posture defines a particular gesture.

We also have described another method which uses previous frame as its context and recognize the gesture performed. Many a times, a gesture recognized can have multiple meaning, or its actual meaning cannot be easily recognized by just extracting features from one frame. So, in such situations we can use previous frame of the gesture performed and extract its features. The information obtained from these features can then be used to avoid the ambiguity in meaning and the gesture can be recognized accurately. These gestures then can be used to make some complex decisions, ultimately driving an application.
\end{abstract}

\section{Keywords:}

Grey scale Manipulation, Smoothing, Edge and feature detection, Face and gesture recognition.

\section{Introduction:-}

A particular relative position of body part is a posture. And posture can be used to convey some information in conversation. But most of time it may happens that the same body posture gives multiple meaning. Then such a posture can be called as the ambiguous posture. In our approach, we try to reduce the ambiguity regarding the meaning of such postures. For reducing the ambiguity we require some extra information. The extra information may be anything related to the posture. In our approach we tried to reduce the ambiguity regarding the series of hand postures. And we used the facial expression and previous frame (posture) as context information to reduce the ambiguity regarding the meaning of the hand posture. e.g. the meaning of hand posture is different for different facial expression. Additionally, the previous frame's posture can be use to reduce the ambiguity of the hand posture. We can link the previous frame's posture with current one to find the complete meaning.

According to our approach first we have to extract the hand posture information from each frame in series of frames. A large number of literatures are reported by the researcher for static hand DOI : $10.5121 /$ cseij.2012.2203 
posture recognition. Mr. Chetan Burande presented one approach for hand gesture recognition [10]. Three stages of the proposed algorithm proposed by Mr. Burande are based on a new hand tracking technique to recognize the actual beginning of a gesture using a Kalman filtering process, hidden Markov models and graph matching. Naidoo and Glaser [11] developed a system that is recognized static hand gesture against complex backgrounds based on South African Sign Language (SASL), a (Support Vector Recognition) system used to classify hand postures as gestures. Similarly, large numbers of literature are reported by researcher of static hand posture recognition [12] [14] [15].

As there is ambiguity regarding the meaning of the hand posture, we extract the facial expression information from the frame. There are lots of approaches for facial expression recognition.

Yuwen Wu Hong Liu present a method of modeling facial expression space for facial expression recognition by fuzzy integral [15]. Similarly various approaches were discussed for facil expression recognition [16] [17]. Priya Metri, Jayshree Ghorpade and Ayesha Butalia presented approach[18] in which facial expression can be used as the contextual information for reducing the ambiguity of hand posture. But still there may be some ambiguity regarding the meaning of the gesture. So we have to use the previous hand posture as contextual information to conclude the exact meaning of the hand posture.

In this paper, we presented an approach for hand posture recognition using segmentation. Then we presented one approach which links the series of hand postures to recognize the exact meaning of it.

\section{Methodology:-}

Our main objective is to reduce the ambiguity of the same gesture using the context i.e. Facial expression and the previous frames. So, we can divide our approach in two main modules.

\subsection{Using facial expressions as context:}

In our approach, the continuous video is given as the input. This video is broken down into number of various static frames. If the conjugative frames are containing the same features then we can eliminates one of them. Then we indentify the independent frames. The independent frame means image consist of the hand and face. After getting all the independent frames we apply the following steps.

\subsubsection{Step 1: Hand Feature Extraction.}

So, first we have to extract the hand feature from the image [1] [2]. We describe the hand posture in the term of the finger raised and the orientation of the hand posture. So the feature vector can be given by,

$\mathrm{F}_{\mathrm{HS}}=\left\{\mathrm{F} 1 \wedge{ }^{\wedge} \mathrm{F} 2 \wedge \mathrm{F} 3{ }^{\wedge} \mathrm{F} 4{ }^{\wedge} \mathrm{F} 5, \mathrm{O}_{\mathrm{R}}\right\}$

Where,

F1 - F5 indicates the positioning of the each finger from little finger to thumb. (i.e. 1 or 0 , raised or not raised)

$\mathrm{O}_{\mathrm{R}}$ indicate the orientation of hand posture. 


\subsubsection{Step 2: Using the facial expressions.}

We can't conclude the meaning of same hand posture in various contexts. To reduce this ambiguity regarding interpretation face feature extraction [3] [5] [7] is done as next step.

The task of automatic facial expression recognition from face image sequences is divided into the following sub problem areas: detecting prominent facial features such as eyes and mouth, representing subtle changes in facial expression as a set of suitable midlevel feature parameters, and interpreting this data in terms of facial gestures. Action units are used as face features. Action unit includes the variation of eyebrows, mouth and chick from original look, showing the reaction.

\subsection{Using previous frames as context:}

Some ambiguities are still remaining though facial expressions are used as a context. To reduce these ambiguities we are using the information from previous frames as a new context. Every state contains information regarding all the considered previous states, next state and corresponding meanings. For that we defined the node structure in such a way that it could store multiple meanings for the same hand posture. Similar procedure is repeated until video get completed.

We are using the concept of tree to elaborate above theme. Consider each node of the tree as one state. And each state represents one hand posture. The state consist of the hand features, face features and possible meanings at that state. Each meaning is associated with previous context information. And also each meaning is associated with next possible postures. Each possibility changes the meaning of the current posture. Therefore, each meaning can be represented by edge in the tree. The edge is selected based on the meaning taken at current state and go to the next state.

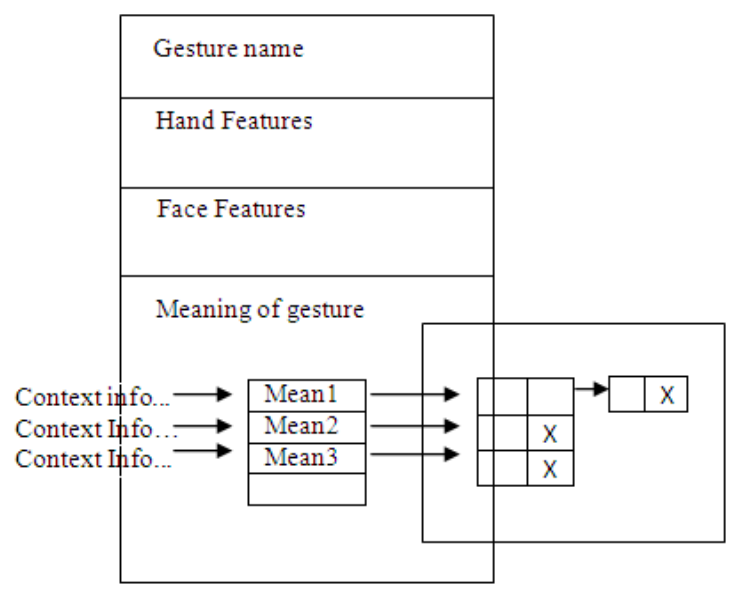

Figure 1: Each state of the tree.

The path consists of various states. At each state it gains more and more contextual information that leads in reduction of ambiguity. We can formulate the meaning at each state as, 


$$
M i=m c+\sum_{p=0}^{i-1} m p
$$

Where, $\mathbf{M}_{\mathrm{i}}$ is meaning of current state, $m p$ is previous states' context information and $m c$ is information within current state.

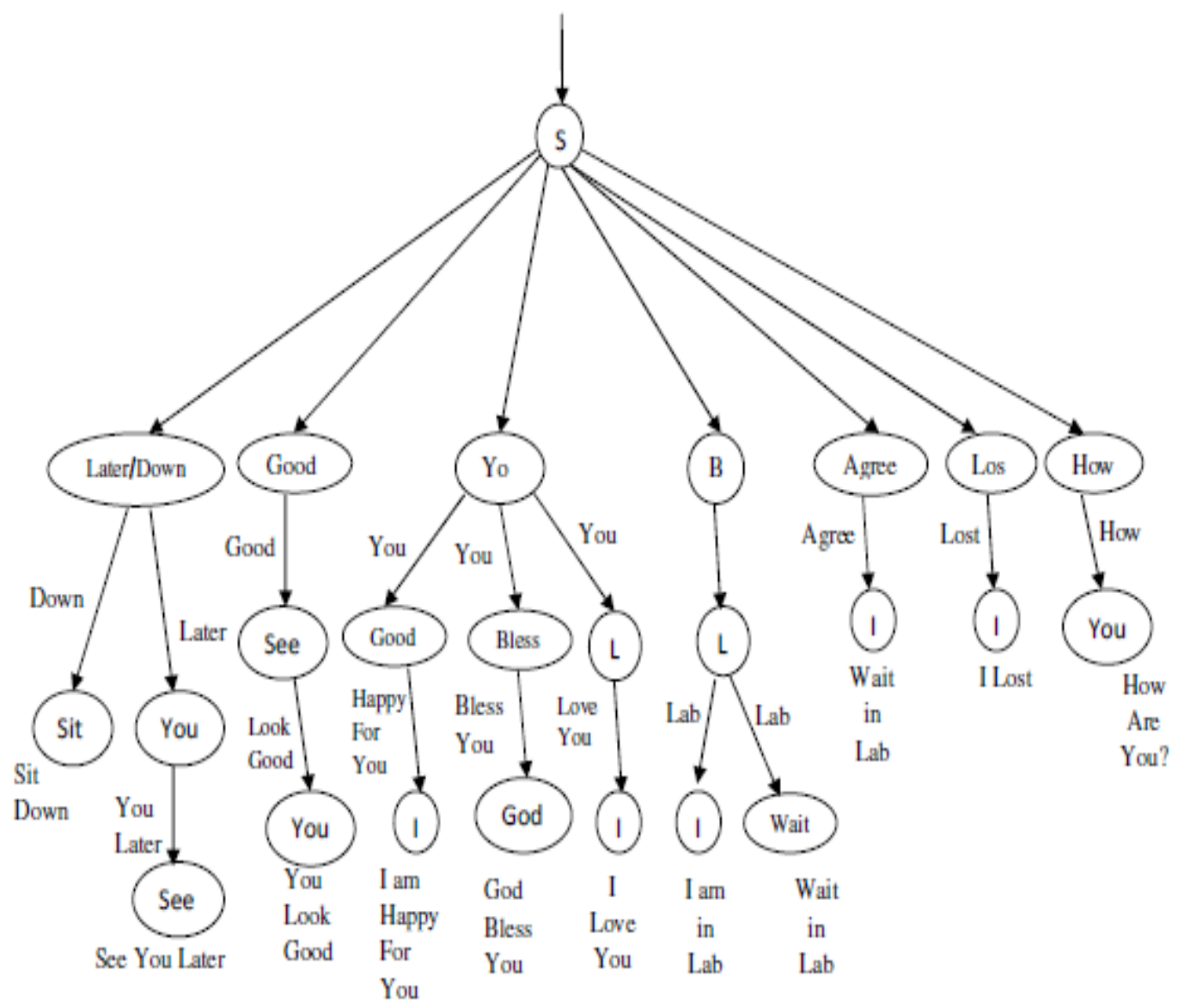

Figure 2: Tree for Dataset 1

Sometimes there is no edge to select then failure is occurred. Failure can be identified in one of the two possible ways.

i. Not Final Stare ${ }^{\wedge}$ Video Over

The video is over and still we are not on the final state.

ii. Final State ${ }^{\wedge}$ Video remains

We are on the final state and still the video remains. 


\section{Case Study:-}

From the various gestures stored in the dataset consider one gesture for an example,

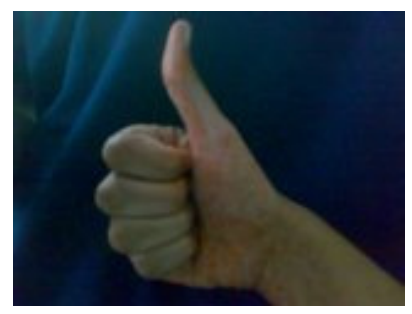

Figure 3: hand image for thumb rose.

The hand feature can be given as,

$\mathrm{F}_{\mathrm{HS}}=\left\{\mathrm{F} 5,90^{\circ} \mathrm{CCW}\right\}$

This can have multiple meaning i.e.

\{All the Best, Ok, Up-direction, God\}

So for reducing the ambiguity in their meaning we have to use facial expression.

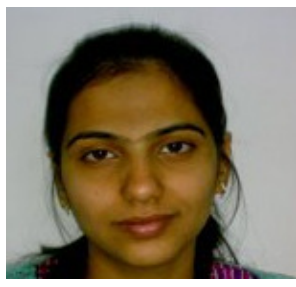

(a)

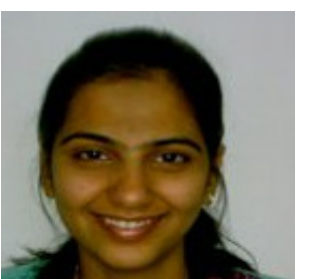

(b)

Figure 4: (a) Normal Facial Expression. (b) Happy facial expression.

Hence the two class formed are,

1) $\{$ All The best, God $\}$

- For happy facial expressions

2) $\{$ Ok, Up Direction $\}$

- For Normal facial expression.

Still there is ambiguity regarding the meaning of the gesture. So we have to use the previous frames as the contextual information. So consider the video contains series gestures for sentences "See you later" and "Sit down".

As we are traversing the video from last to first there is ambiguity at the first stage because the word "later" and "down" has same gesture. So we have to check for the previous frame information. If previous frame features matches with those of "YOU" then it will be considered as "later". If it matches with "SIT", then consider the meaning as "down". Else consider it as failure. 


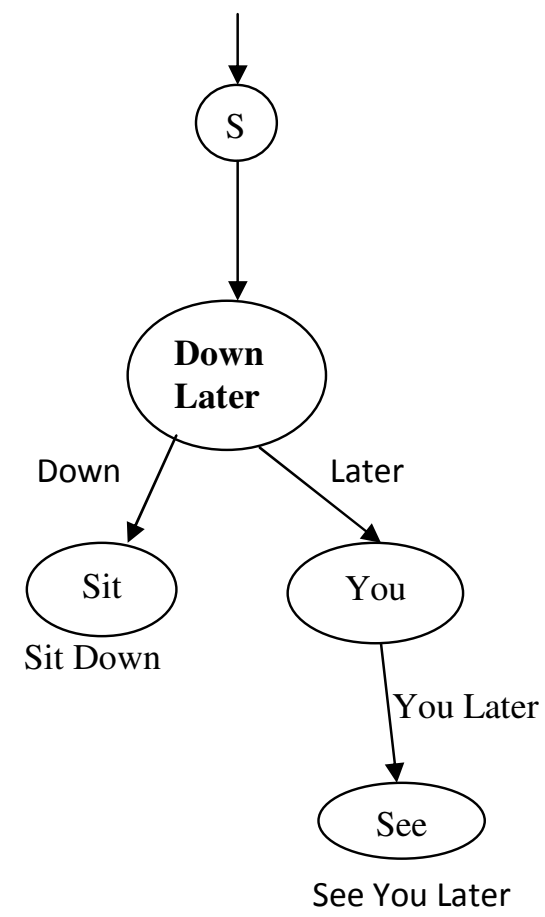

Figure 5: Tree traversal for extracting the meaning of conflicting gesture (down/later).

\section{Experimental Results:}

\begin{tabular}{|c|c|c|}
\hline & $\begin{array}{l}\text { Recognition } \\
\text { rate for using } \\
\text { Only Face as } \\
\text { context. }\end{array}$ & $\begin{array}{l}\text { Recognition } \\
\text { rate for using } \\
\text { previous frame } \\
\text { as context }\end{array}$ \\
\hline Dataset 1 & $67.4 \%$ & $84.4 \%$ \\
\hline Dataset 2 & $63.2 \%$ & $85.6 \%$ \\
\hline Dataset 3 & $61.3 \%$ & $82.1 \%$ \\
\hline
\end{tabular}

Table 1: Experimental results.

For experimenting, the results of our system we tested our system on three of the datasets. Those datasets consists of various approx. 20-25 sentences each. Each sentence consists of series of 3-4 gestures. These results show the rate of recognition of the gesture using facial expression as context and using the previous frame as context. 
Computer Science \& Engineering: An International Journal (CSEIJ), Vol.2, No.2, April 2012

\section{Conclusion:-}

The methods described above can be effectively used to accurately determine the meanings of gestures performed. In the techniques discussed till now gestures are recognized using different contexts as windows applications, etc. Here facial expressions are an easy way to be used as extra information to remove the ambiguity in the meanings of gestures performed. A lot of other information can be used as context in gesture recognition. For example, environment variables, applications active on operating system, etc.

This system can have many different applications. It can be used in robotics. This can be used as an intermediate system in communication process in different applications. It can be used by the dumb people to communicate with different systems as an easy way of interaction.

\section{References:}

[1] Elena Sánchez-Nielsen, Luis Antón-Canalís, Mario Hernández-Tejera, "Hand Gesture Recognition for Human-Machine Interaction".

[2] Hironori Takimoto, Seiki Yoshimori, Yasue Mitsukura and Minoru Fukumi, "Classification of Hand Postures Based on 3D Vision Model for Human-Robot Interaction", 19th IEEE International Symposium on Robot and Human Interactive Communication, Italy. Sept. 12-15, 2010.

[3] P.Pramod Kumar, Prahlad Vadakkepat, and Loh AI POH,"Hand Posture and face recognition using fuzzy rough approach", International Journal of Humanoid Robotics Vol. 7, No. 3 (2010).

[4] B. Stenger P. R. S. Mendonc, a R. Cipolla, "Model-Based 3D Tracking of an Articulated Hand" , In proc. British Machine Vision Conference, Manchester, UK, September 2001.

[5] M. Pantic, L.J.M. Rothkrantz, "Facial gesture Recognition in face image sequences: A study on facial gestures typical for speech articulation”, Delft University of Technology.

[6] Bernard Boulay, Francois Bremond, Monique Thonnat, "Human Posture Recognition in Video Sequence", INRIA Sophia Antipolis, France.

[7] Balasubramani A, Kalaivanan K, Karpagalakshmi RC, Monikandan R, "AUTOMATIC FACIAL EXPRESSION RECOGNITION SYSTEM”, International Conference on Computing, Communication and Networking 2008.

[8] Michael Kipp, Jean-Claude Martin, "Gesture and Emotion: Can basic gestural form features discriminate emotions?", Affective Computing and Intelligent Interaction and Workshops, 2009. ACII 2009. 3rd International Conference on 10-12 Sept. 2009.

[9] Jun Ou, Xiao-Bo Bai, Yun Pei ,Liang Ma, Wei Liu, “Automatic Facial Expression Recognition Using Gabor Filter And Expression Analysis”, Second International Conference on Computer Modeling and Simulation 2010.

[10] Mr. Chetan A. Burande, Prof. Raju M. Tugnayat, Prof.Dr. Nitin K. Choudhary, "Advanced Recognition Techniques for Human Computer Interaction”, Computer and Automation Engineering (ICCAE), 2010 The 2nd International Conference on 26-28 Feb. 2010.

[11] S. Naidoo, C. Omlin and M. Glaser, "Vision-Based Static Hand Gesture Recognition Using Support Vector Machines", 1998.

[12] Ghosh, Dipak Kumar, "A static hand gesture recognition algorithm using k-mean based radial basis function neural network", Information, Communications and Signal Processing (ICICS) 2011 8th International Conference on 13-16 Dec. 2011.

[13] Igorevich, R.R.Sch. of Comput. Sci. \& Eng., Konkuk Univ., Seoul, South Korea Pusik Park ; Dugki Min ; Yunjung Park ; Jongchan Choi ; Eunmi Choi,” Hand gesture recognition algorithm based on grayscale histogram of the image", Application of Information and Communication Technologies (AICT), 2010 4th International Conference on 12-14 Oct. 2010

[14] Tofighi, G. Univ. of Isfahan, Isfahan, "Rapid hand posture recognition using Adaptive Histogram Template of Skin and hand edge contour", Machine Vision and Image Processing (MVIP), 2010 6th Iranian, 27-28 Oct. 2010.

[15] Yuwen Wu, "Modeling facial expression space for recognition", Intelligent Robots and Systems, 2005. (IROS 2005). 2005 IEEE/RSJ International Conference on 2-6 Aug. 2005 
Computer Science \& Engineering: An International Journal (CSEIJ), Vol.2, No.2, April 2012

[16] Yu-Li Xue Sch. of Electron. \& Inf. Eng., Beihang Univ. Xia Mao ; Fan Zhang,” Beihang University Facial Expression Database and Multiple Facial Expression Recognition”, Machine Learning and Cybernetics, 2006 International Conference on 13-16 Aug. 2006.

[17] Aleksic, P.S. Electr. Eng. \& Comput. Sci. Dept., Northwestern Univ., Evanston, IL, USA Katsaggelos, A.K.,"Automatic facial expression recognition using facial animation parameters and multistream HMMs", Information Forensics and Security, IEEE Transactions on March 2006.

[18] Priya Metri, Jayshree Ghorpade and Ayesha Butalia, "Facial Emotion Recognition Using Context Based Multimodal Approach”, Int. J. Emerg. Sci., 2(1), 171-182, March 2012 ISSN: 2222-4254

\section{Authors}

Ayesha Butalia She is pursuing PhD from Devi Ahilya University, Indore. She has been the University Topper in Masters for Computer Science and Engineering, specialization in Information Technology from VIT, Pune in 2008. She holds Junior Diploma in French language from Nagpur University. She also holds Certification of IBM-ACE, DB2, RSA, Rational Rose and many more. She is currently working as a Associate Professor in Maharashtra Institute of Technology, College of Engineering, Pune.

Maya Ingle Presently, working as Professor/ Senior System Analyst, School of Computer Science and Information Technology, D.A. University for past 8 years. Also, worked as Dean, Student Welfare, Devi Ahilya University, Indore from Aug. 2006 to Sept. 2009. Worked as Principal, Indore Institute of Science and Technology, Indore. (M.P.) Indiafrom 4 Sept'09 to 2 Sept'11. Working Experience: Around 26 years of Technical and Administrative experience. Visiting Professor at I.I.I.T. Jabalpur and I.I.T. Indore. Major Research Areas of Interest: Software Engineering, Statistical Natural Language. 